\title{
ABSTENCIONISMO ELECTORAL EN CONTEXTOS NO DEMOCRATICOS Y DE TRANSICION: EL CASO ESPAÑOL
}

Luis López Guerra

\section{INTRODUCCION}

El estudio de los resultados de consultas electorales se ha revelado como un medio óptimo para descubrir y analizar tensiones políticas existentes entre los sectores sociales que integran una comunidad, sean clases, regiones o grupos religiosos: el análisis de tales cleavages, por utilizar el término anglosajón, ha encontrado en la investigación electoral su arma natural. Ahora bien, este tipo de estudios, a la búsqueda de tensiones políticas, se ha centrado casi en régimen de exclusividad en el análisis de los regímenes democrático-liberales. Lo que, obviamente, no es de extrañar, si consideramos que todo el proceso electoral es significativo solamente si se dan tres condiciones mínimas: por una parte, que exista una garantía de que los resultados que se hacen públicos son los efectivamente expresados, esto es, que no haya una falsificación de cifras electorales; en segundo lugar, que la expresión de la voluntad popular se efectúe libremente, sin coacciones que invaliden o hagan desaparecer la voluntad real del elector, y, finalmente, que éste se pronuncie, en elecciones o referendos, sobre auténticas alternativas, que compitan libremente entre sí. Veracidad en los resultados, libertad en la emisión del voto y competición entre fuerzas políticas en condiciones de igualdad se configuran, así, como los requisitos teóricos sine qua non, para aceptar los 
resultados electorales como indicadores de las actitudes políticas de los componentes de una comunidad, dotados al menos de validez aparente.

Así y todo, y manteniendo in mente tal principio, es posible conjeturar que, a falta de otros indicadores, resultados electorales que no cumplan $\longrightarrow$ cumplan imperfectamente- tales requisitos, pueden resultar útiles a la hora de determinar algunas características políticas de regímenes que carecen de elecciones libres, o en los que éstas han sido pocas. Los resultados de consultas electorales o referendarias, en países no-democráticos (en el sentido usual del término), podrían quizá facilitar importantes datos sobre corrientes de opinión, distribución de actitudes políticas y otros fenómenos difíciles de conocer de otro modo, ante la ausencia o falta de fiabilidad de otras fuentes. Siempre, desde luego, teniendo presente el vicio de origen de las cifras obtenidas en tales contextos.

Así, contamos con algunos estudios sobre comportamiento electoral en contextos autoritarios: en los países socialistas ${ }^{1}$ y en el caso español entre 1947 y $1976^{2}$. En estos casos, desde luego, no se daba una competición electoral (ya que las elecciones de que se trata han sido, bien sin partidos, como en el caso español de 1967 y 1971 en las elecciones a procuradores «por el tercio familiar», bien con un partido único, como en el caso soviético, o bien, al menos, con una lista única, como en otros países socialistas) ni, si se trataba de referendos, se daban alternativas claras a la posición oficial, ni posibilidad de propaganda en contra de ésta, ni, finalmente, un régimen de libertades públicas que garantizase la independencia del elector, ni, al menos en el caso español, la fiabilidad de las cifras oficialmente publicadas ${ }^{3}$.

Sin embargo, y si tenemos en cuenta tales cifras oficiales, nos encontramos de inmediato con el hecho curioso de que presentan un considerable rango de variaçión, tanto si comparamos unos países con otros como si comparamos cifras dentro del mismo país en zonas y ocasiones diferentes. Diferencias que se refieren sobre todo al grado de participación electoral, y que muestran que, incluso en regímenes no democráticos, cabría utilizar las cifras oficiales para llevar a cabo cierto análisis de sus condiciones políticas y

1 Por ejemplo, F. Dinka y M. SKmmore, "The Functions of Communist One Party Elections: Czechoslovakia 1971", en Political Science Quarterly, septiembre 1973; J. Gilison, "Soviet Elections as a Measure of Dissent: The Missing One per Cent", American Political Science Review, septiembre 1968, págs. 814-826.

2 Véanse, entre los más recientes, Luis AguIAR DE LUQUe, Democracia directa y Estado constitucional, Ed. Revista de Derecho Privado, Madrid, 1977, págs. 274-308, y L. Aguiar y M. Aragón, "El referéndum de 15 de diciembre de 1976 en Madrid", Revista Española de la Opinión Pública, abril-junio de 1977, págs. 115-156. También los diversos trabajós al respecto en el número "Las elecciones en España", de Historia 16, de abril de 1977, así como la bibliografía que cita el autor de estas lineas en su trabajo "La perspectiva actual: algunas notas sobre el caso español", en J. DE Esteban (ed.), El proceso electoral, Labor, Barcelona, 1977, págs. 316-344, referida a autores como Martf́nez Cuadrado, Molas, Hramet y otros.

${ }_{3}^{3}$ Véase V. Agular, Democracia directa..., op. cit., págs. 280 y sigs. 
posibles tensiones, tomando en cuenta no la dirección (o contenido concreto) del voto en elecciones y referendos, sino la mayor o menor abstención por parte de los electores. Y ello en dos formas: estudiando las diferencias en participación entre países y dentro de cada país (en contextos autoritarios), por un lado; y comparando, cuando ello sea posible, el abstencionismo electoral en consultas democráticas y no democráticas dentro del mismo país, por otro.

\section{LAS DIFERENCIAS EN PARTICIPACION}

En la moderna literatura sobre elecciones ha ido aumentando el interés por el fenómeno del abstencionismo electoral, interés que en ocasiones ha ido unido a la consideración de que el valor de las elecciones es más de apoyo al sistema, o a un grupo dentro del mismo, que de selección de soluciones políticas: «probablemente sería mejor considerar que las elecciones no tienen mucho que ver con soluciones específicas, y que no hacen sino conceder a un grupo gobernante algo así como una aprobación general a su programa» ${ }^{4}$. Aun sin llegar al extremo de desdeñar los resultados electorales concretos, se ha señalado que el mero hecho de participar o no en una elección o referéndum ya supone cierta indicación de la postura política de un sujeto con respecto al régimen en cuestión, por cuanto que la mera participación supone, bien una aceptación de la legitimidad del sistema, bien, más crudamente, el reconocimiento de su capacidad de coacción caso de abstención del ciudadano del proceso electoral. Tanto en el caso de convencimiento de la legitimidad del sistema, como de miedo ante el poder coactivo del mismo, el poder gozará de una capacidad de movilización y control de la población ${ }^{5}$.

El problema del abstencionismo se plantea, pues, en regímenes democráticos y autoritarios, si bien desde perspectivas distintas: para una democracia, la abstención puede suponer la existencia de corrientes políticas que no se integran en el juego político normal: «... una sociedad en que una amplia proporción de la población queda fuera de la arena política es potencialmente más explosiva que una sociedad en la que la mayoría de los ciudadanos toma parte regularmente en actividades que les dan cierto sentimiento de participación en las decisiones que afectan a sus vidas» ${ }^{6}$. Para un régimen autoritario, el abstencionismo puede significar la incapacidad para llevar a cabo una movilización (y coacción) efectiva de la población, y, a veces, el no poder confiar siquiera en los mismos resortes del poder, que habrian de hacer posible la manipulación de los electores o de los votos.

4 R. Dowse y J. R. Hughes, Sociología politica, Alianza, Madrid, 1975, pág. 406.

5 Véanse GILISON, op. cit., y A. J. Milnor, Elections and Political Stability, Little Brown, Boston, 1969.

${ }^{6}$ LIPSET, S. M., Political Man, Doubleday, Garden City, 1963, pág. 184. 
La pregunta a plantearse, pues, ante las consultas electorales o referendarias en contextos autoritarios sería la referente no a cuáles son las alternativas en presencia (irrelevantes ante una victoria gubernamental conocida de antemano), ni su respectivo peso electoral (probablemente falsificado), sino relativas a la capacidad del sistema para movilizar al electorado, bien mediante la convicción, bien mediante la coacción.

¿Es tal capacidad alta -el régimen puede movilizar a la gran mayoríao baja -el régimen sólo puede movilizar a unos cuantos-? ¿Es una capacidad de movilización uniforme -igual en todos los distritos o regiones de un país- o variable, según las diversas regiones? En lo que se refiere al primer aspecto, la utilidad de estas comparaciones entre diversos tipos de regímenes no democráticos puede comprobarse echando una ojeada a los resultados de elecciones y referendos llevados a cabo en diversos contextos. Comparando tales resultados, parece plausible deducir ciertas diferencias en su capacidad movilizadora, diferencias plasmadas en la distinta afluencia de votantes que cada régimen puede conseguir en unas circunstancias en que no existen, desde luego, posibilidades de un resultado desfavorable al poder (y el electorado es consciente de ello). Claramente, viendo los diversos resultados expresados en la tabla I, uno no puede por menos de preguntarse si las diferencias expuestas no representan la existencia de verdaderas diferencias entre regímenes.

\section{TABLA I}

Consultas electorales y referéndum en contextos no democráticos ${ }^{7}$

\begin{tabular}{|c|c|c|}
\hline País y tipo de consulta & Año & $\begin{array}{l}\text { Porcentaje de } \\
\text { participación } \\
\text { sobre el censo }\end{array}$ \\
\hline URSS, elecciones al Soviet de la Unión ... ...... & 1950 & 99,98 \\
\hline AUSTRIA, referéndum Anschluss $\ldots \ldots \ldots \ldots \ldots$ & 1938 & 99,71 \\
\hline 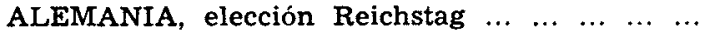 & 1938 & 99,60 \\
\hline 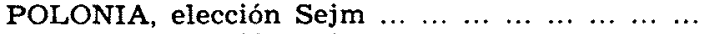 & 1952 & $\mathbf{9 5 , 3 0}$ \\
\hline POLONIA, elección $\operatorname{Sejm} \ldots \ldots \ldots \ldots$ & 1957 & 94,10 \\
\hline $\begin{array}{llllllllll}\text { ESPANA, referéndum } & \ldots & \ldots & \ldots & \ldots & \ldots & \ldots & \ldots & \ldots & \ldots\end{array}$ & 1966 & 89,19 \\
\hline 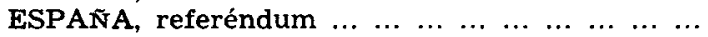 & 1976 & 77,72 \\
\hline 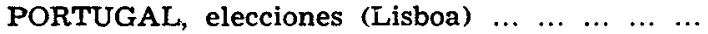 & 1969 & 48,10 \\
\hline
\end{tabular}

7 Fuentes: Para la URSS, Ellen Mickrewicz, Handbook of Soviet Social Science Data, Free Press, Nueva York, 1973, pág. 170. Para Austria y Alemania, Statistiches Jahrbuch für das Deutsche Reich, 1938, pág. 618. Para Polonia, D. Butler, Elections Abroad, Mac Millan, Londres, 1959. Para España, datos de la Junta Central del Censo. Para Portugal, Ruiz Garcfa, "Las elecciones de Portugal, cara y cruz de un comportamiento político", Cuadernos para el Diálogo, noviembre 1969, págs. 28-34. 
No dejan de ser indicativas estas cifras a la hora de mostrar los diferentes tipos de regímenes autoritarios que eran los constituidos por el nacional-socialismo alemán y el socialismo soviético de 1950, basados en una supermovilización de las masas, y por otro lado, el caso portugués, basado en una inframovilización, esto es, en la apatía política voluntariamente bus. cada por el poder. España ocupa un lugar intermedio, siendo sus tasas de participación en 1966 y 1976 inferiores no sólo a las de otras dictaduras, sino incluso menores que las tasas de participación de países democráticoliberales, como la República Federal Alemana $(91,1$ por 100 de participación electoral en las elecciones de $1972^{8}$ ).

Es posible, desde luego, afinar más. Pues, en efecto, nos hemos referido, en el cuadro anterior, a diferencias entre países, en sus cifras de participación. Pero podemos ir más allá y hacer comparaciones dentro de cada país, comparaciones de orden espacial o temporal, a efectos de encontrar diferencias que pueden quedar ocultas por la cifra de participación global. ¿Es uniforme la capacidad de movilización electoral del régimen o varía geográficamente? $\mathrm{Y}$ si es este último el caso, ¿qué conclusiones pueden deducirse? Podemos elegir, para mostrar casos concretos, tres ejemplos: la Alemania nacional-socialista, la Polonia del «deshielo» y la España del primer referéndum (Tabla II).

\section{TABLA II}

\section{Diferencias en participación electoral en contextos no democráticos 9}

\begin{tabular}{|c|c|c|c|c|}
\hline Pais y tipo de elección & $A \bar{n} o$ & \% participación & $\%$ & $\begin{array}{c}\text { Variación } \\
(\%)\end{array}$ \\
\hline Alemania, referéndum $19 \ldots$ & 1938 & $\begin{array}{c}\text { Máximo } \\
\text { (Rhein Pfalz) } \\
\text { Mínimo } \\
\text { (Weser Ems) }\end{array}$ & $\begin{array}{l}99,91 \\
97,87\end{array}$ & 2,04 \\
\hline Polonia, elección Sejm ... ... & 1957 & $\begin{array}{c}\text { Máximo } \\
\text { (Bialystok) } \\
\text { Mínimo } \\
\text { (Tychy-Katowice) }\end{array}$ & $\begin{array}{l}97,00 \\
80,60\end{array}$ & 16,40 \\
\hline España, referéndum $\ldots \ldots \ldots$ & 1947 & $\begin{array}{l}\text { Máximo } \\
\text { (Valladolid) } \\
\text { Minimo } \\
\text { (Oviedo) }\end{array}$ & $\begin{array}{r}96,98 \\
76,85\end{array}$ & 20,13 \\
\hline
\end{tabular}

${ }^{8}$ Véanse las cifras en T. MAckie y R. Rose, The International Almanac of Electoral History, Mac Millan, Londres, 1974, págs. 162-163.

9 Fuentes: Mismas que en nota 7, y Anuario Estadistico, 1948. 
Estas cifras no dejan de mostrar la diferente naturaleza de los regímenes en cuestión. Frente al monolitismo del Tercer Reich (sin apenas diferencias en la respuesta electoral), la Polonia inmediatamente posterior a la crisis posstalinista muestra una mayor diferenciación intranacional, quizá debida a tensiones políticas en la sociedad polaca: diferenciación que se acentúa aún más en la España de 1947, en situación de bloqueo internacional, y dominada aún por el fantasma, frecuentemente enarbolado, de la guerra civil.

\section{EL CASO ESPAÑOL}

Cabe por tanto, examinar los resultados de las consultas electorales y referendarias españolas en la etapa 1947-1976, al objeto de determinar si existe un grado de variación interprovincial que pueda proporcionar datos sobre el arraigo de tensiones políticas durante la época; y, por otro lado, pudiera ser útil verificar si una comparación de los resultados de estas consultas con los derivados de las elecciones de 1977 (en un contexto político diferente) viene a ratificar de alguna manera la permanencia de tales tensiones.

Por lo que se refiere al primer punto, la variación interprovincial, nos encontramos con un amplio rango de variación (Tabla III).

\section{TABLA III}

Diferencias de participación electoral entre provincias ${ }^{10}$

\begin{tabular}{|c|c|c|c|c|}
\hline Tipo de consulta & Año & Participación & & Variación \\
\hline $\begin{array}{llllll}\text { Referéndum } & \ldots & \ldots & \ldots & \ldots & \ldots\end{array}$ & 1947 & $\begin{array}{c}\text { Máximo } \\
\text { (Valladolid) } \\
\text { Mínimo } \\
\text { (Oviedo) }\end{array}$ & $\begin{array}{l}96,98 \\
76,85\end{array}$ & 20,13 \\
\hline $\begin{array}{llllll}\text { Referéndum } & \ldots & \ldots & \ldots & \ldots & \ldots\end{array}$ & 1966 & $\begin{array}{l}\text { Máximo } \\
\text { (Baleares) } \\
\text { Mínimo } \\
\text { (Guipúzcoa) }\end{array}$ & $\begin{array}{l}98,04 \\
75,83\end{array}$ & 22,21 \\
\hline Elecciones procuradores... ... & 1967 & $\begin{array}{l}\text { Máximo } \\
\text { (Teruel) } \\
\text { Mínimo } \\
\text { (Guipúzcoa) }\end{array}$ & $\begin{array}{l}84,68 \\
36,64\end{array}$ & 48,04 \\
\hline Elecciones procuradores... ... & 1971 & $\begin{array}{c}\text { Máximo } \\
\text { (Logroño) } \\
\text { Múnimo } \\
\text { (Guipúzcoa) }\end{array}$ & $\begin{array}{l}79,90 \\
26,00\end{array}$ & 53,90 \\
\hline $\begin{array}{llllll}\text { Referéndum } & \ldots & \ldots & \ldots & \ldots & \ldots\end{array}$ & 1976 & $\begin{array}{l}\text { Máximo } \\
\text { (Logroño) } \\
\text { Mínimo } \\
\text { (Guipúzcoa) }\end{array}$ & $\begin{array}{l}87,15 \\
45,25\end{array}$ & 41,90 \\
\hline
\end{tabular}

${ }^{10}$ Fuentes: Mismas que en notas 7 y 9. 
La pregunta inmediata que se plantea, pues, es el porqué de tal variación. Aun dejando de lado, en efecto, los resultados de las elecciones a procuradores en Cortes de 1967 y 1971, en las que el aparato estatal no parece haberse volcado tan a fondo como en 1966 y 1947, el rango de variación aparece como muy superior al acostumbrado en regímenes autoritarios.

Cabría aducir que se trata de cifras sin ningún valor representativo, mera invención de las autoridades. Sin embargo, y sin tratar, desde luego, de defender su exactitud, cabe aceptar que, sin tratarse de datos válidos, sí pueden considerarse en parte indicativos, al menos en lo que se refiere a las causas subyacentes en su variación. Por ejemplo, seleccionando en los tres referendos de 1947, 1966 y 1976, las diez provincias con mayor índice de abstenciones, nos encontramos con considerables coincidencias (Tabla IV).

\section{TABLA IV}

Provincias con mayor indice de abstención ${ }^{11}$

\begin{tabular}{|c|c|c|}
\hline 1947 & 1966 & 1976 \\
\hline $\begin{array}{l}\text { Oviedo } \\
\text { Lugo } \\
\text { Tenerife } \\
\text { Pontevedra } \\
\text { Barcelona } \\
\text { Coruña, La }\end{array}$ & $\begin{array}{l}\begin{array}{l}\text { Oviedo } \\
\text { Lugo } \\
\text { Tenerife }\end{array} \\
\\
\text { Guipúzcoa } \\
\text { Vizcaya } \\
\text { Orense } \\
\text { Zamora } \\
\text { Sevilla } \\
\text { Palencia } \\
\text { Avila }\end{array}$ & $\begin{array}{l}\text { Oviedo } \\
\text { Lugo } \\
\text { Tenerife } \\
\text { Pontevedra } \\
\text { Barcelona } \\
\text { Coruña, La } \\
\text { Guipúzcoa } \\
\text { Vizcaya } \\
\text { Orense } \\
\text { Navarra }\end{array}$ \\
\hline
\end{tabular}

De los treinta puestos de la tabla, veintitrés están ocupados por provincias que figuran en más de una ocasión entre las diez con más abstención; tres de ellas (Oviedo, Lugo y Tenerife) figuran en tres ocasiones; otras siete (Pontevedra, Barcelona, La Coruña, Orense, Vizcaya, Guipúzcoa y Zamora) en dos ocasiones. Si bien la coincidencia no es desde luego perfecta, parece excluir que las cifras oficiales sean meramente aleatorias (por ejemplo, la posibilidad de que una provincia entre cincuenta figure en tres oca-

1 El orden de las provincias corresponde sólo a efectos de mayor claridad expositiva. 
siones es de $1 / 125$, y hay tres provincias en tal situación). Aceptando, pues, un cierto carácter indicativo en estas cifras, queda el explicar las posibles causas de este abstencionismo y las tensiones políticas que refleja.

Ahora bien, las explicaciones dadas al abstencionismo electoral son múltiples ${ }^{12}$ :

- Algún autor, como Tingsten ${ }^{13}$, o Lipset ${ }^{14}$, ha podido ver la causa del abstencionismo electoral en una confianza implícita en el sistema político, de tal manera que una extrema afluencia de votantes a las urnas sería más una muestra de crisis que de buena salud política de la comunidad. Así, Lipset ha podido correlacionar la disminución de conflictos sociales en Europa Occidental, en la década de los cincuenta, con una disminución de la participación política. Pero tal no parece ser en modo alguno la causa del abstencionismo en el caso español de 1947-76, en que se ponía el énfasis en la obligatoriedad del voto, y se identificaba, precisamente; abstencionismo con oposición al Régimen.

- Más extendida y plausible sería la postura que correlaciona la participación electoral con factores sociológicos relativos sobre todos al status y la clase social: postura que pone el acento en variables como la edad, el sexo, nivel de educación, nivel de ingresos, etc.

- En tercer lugar se ha señalado la importancia, a la hora de determinar las causas de la participación electoral, de la acción de los grupos políticos organizados (mediante agitación y propaganda electoral, por ejemplo). En el caso español de que tratamos, tal acción política se referiría esencialmente a la propaganda oficial, al ser ilegales los grupos políticos, y al prohibirse de hecho a la prensa la propaganda contraria a la postura gubernamental ${ }^{15}$.

- Cabe, por fin, considerar que en ciertos casos la abstención no es consecuencia de la apatía política (sea ésta. debida a satisfacción con el sistema, a razones socioeconómicas o a falta de estímulos por parte de los actores políticos), sino que, por el contrario, el abstencionismo sería expresión de un alto interés político, y, al mismo tiempo, de una actitud de rechazo del sistema, negándose amplias capas sociales a participar en el juego electoral legal. Algunos autores han considerado que en España la abstención en las elecciones de la época 1947-76 podría considerarse como un síntoma de la falta de apoyo al régimen ${ }^{16}$. Como ejemplo de esta posición de "abstencionismo de rechazo» cita Seymour M. Lipset el caso de la abstención de los anarquistas en las elecciones republicanas de 1933, abstención motivada

12 Nos remitimos, en general, al libro de A. LaNCELot L'abstentionisme électoral en France, Colin, París, 1968.

${ }_{13} \mathrm{H}$. Tingsten, Political Behaviour: Studies in Election Statistics, Bedminster Press, Totowa, 1937.

${ }^{14}$ Political Man, op. cit., cap. 6.

15 Véase Aguiar, Democracia..., loc. cit.

16 Así, Martínez Cuadrado y Guy Hermet. Véase nota 2. 
no por la apatía política, sino, por el contrario, por una posición de interés activo.

W. Flanigan ${ }^{17}$ ha elaborado un interesante esquema que conjunta estas diversas posiciones. En un gráfico, sitúa en el eje horizontal la dimensión «nivel de participación» (bajo, medio o alto), y en el eje vertical la dimensión «nivel de interés» (bajo, medio o alto: figura 1).

Podríamos, pues, diferenciar cuatro casos distintos, según este esquema:

I. Bajo interés-baja participación. Debido a condiciones socioeconómicas, o a falta de incidencia de los actores políticos, el nivel de interés por la política es bajo, lo que se traduce en una escasa participación: este tipo recordaría la «cultura parroquial» expuesta por Almond y Verba. Pero también podríamos incluir en este tipo los casos de abstencionismo «por satisfacción» a que se refería Tingsten.

NIVEL DE

INTERES

POLITICO

Figura 1. Niveles de interés y participación (según Flanigan)

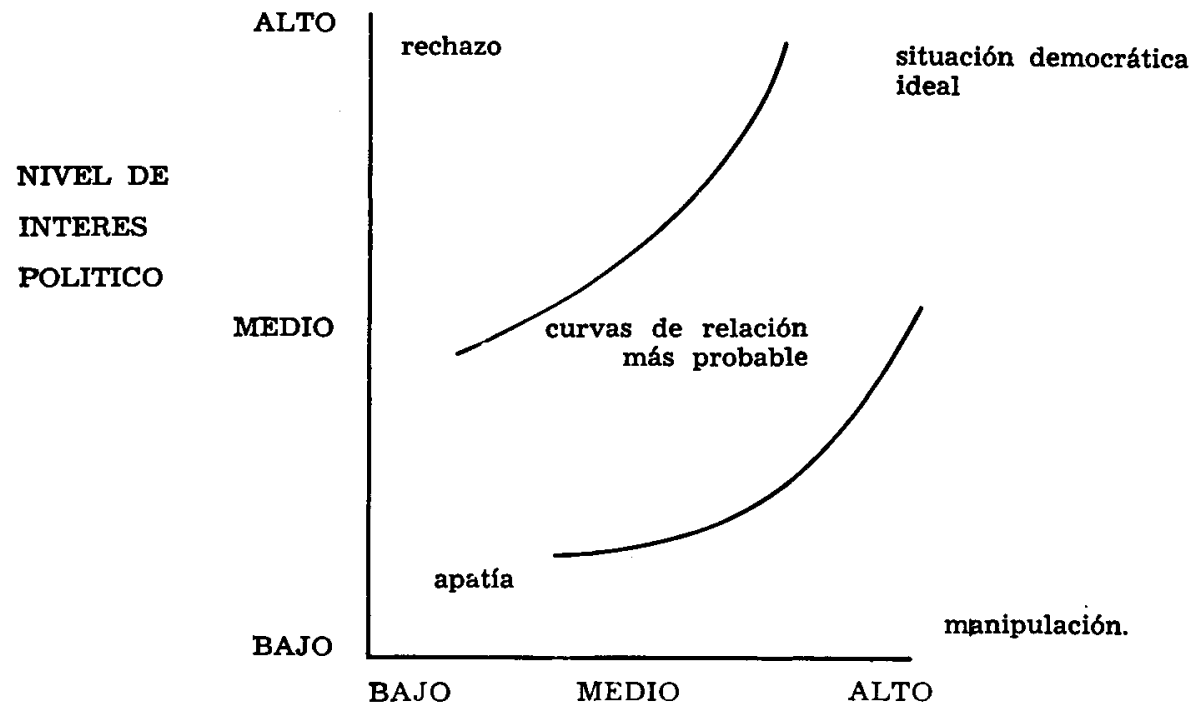

NIVEL DE PARTICIPACION

II. Bajo interés-alta participación. Sería la situación a que se refiere Flanigan con el término de «manipulación»: un electorado sin interés se vería forzado a participar en el proceso electoral, para evitar las sanciones impuestas por el poder en caso contrario. Correspondería, siguiendo el paralelismo anterior, a la «cultura política de sujeción».

${ }^{17}$ Political Behavior of American Electorate, Allyn and Bacon, Boston, 1972. 
III. Alto interés-baja participación. Esta situación es la descrita por Lancelot y Lipset; situación en que, a diferencia del caso I, la baja participación electoral se debería no a una falta de sensibilización política, sino, por el contrario, a una posición política determinada, la de rechazo consciente del sistema político.

IV. Finalmente, la situación que podríamos llamar «ideal» (dejando de lado las advertencias contra una «excesiva» participación, que parecen teñidas de un cierto conservadurismo) en la que coincidirían alto interés político y una elevada participación electoral.

\section{RAZONES DEL ABSTENCIONISMO EN ESPAÑA}

La clasificación de Flanigan resulta aplicable al caso español al permitirnos diferenciar, dentro del abstencionismo, dos posibilidades: la correspondiente al caso I (esto es, abstencionismo debido a apatía política, falta de interés por la vida política, cualquiera que sea el régimen del momento) y la correspondiente al caso III, en la que el abstencionismo se debe, por el contrario, a un alto interés político, traducido en una posición de rechazo consciente del sistema.

Una forma posible de discriminar entre ambas variedades de abstencionismo pudiera ser la de buscar los correlatos más usuales con los diversos tipos de cultura política y mantener la hipótesis de que, por ejemplo, las provincias con bajo nivel de ingreso medio, baja alfabetización, alto índice de dispersión de la población, etc., darían lugar a un abstencionismo «apático» del tipo I, mientras que en contextos industrializados con un alto nivel medio de educación, ingresos, urbanización, etc., el abstencionismo sería más bien del tipo III (rechazo político). Sin embargo, tal solución presenta considerables dificultades, dado que, por ejemplo, las diez provincias con mayor abstencionismo que hemos utilizado en la Tabla IV no se reparten rígidamente en dos grupos (que podríamos llamar desarrolladas y subdesarrolladas), sino que, en el rango de valores de los diversos indicadores utilizables, se reparten todo a lo largo del rango de dispersión de las cincuenta provincias.

En la Tabla V mostramos tal fenómeno de dispersión, tomando en cuenta las diez provincias con mayor nivel de abstención en 1976 , y su posición ordinal en varias listas de indicadores respecto a las demás provincias: renta familiar disponible per capita (1973), tasa de población urbana y porcentaje de la población activa agraria sobre la población activa total (Tabla V). 


\section{TABLA V}

Posición ordinal, entre las cincuenta provincias españolas, de las diez provincias con mayor abstencionismo

en el Referéndum de 1976, respecto

a varios indicadores ${ }^{18}$

Indicadores $y$ orden de posición

\begin{tabular}{|c|c|c|c|}
\hline \multirow[b]{3}{*}{ Provincias } & \multirow{2}{*}{\multicolumn{2}{|c|}{$\sin$}} & \\
\hline & & & Porcentaje \\
\hline & $\begin{array}{c}\text { Renta familiar } \\
\text { disponible } \\
\text { per capita }\end{array}$ & $\begin{array}{l}\text { Tasas de po- } \\
\text { blación urbana }\end{array}$ & $\begin{array}{c}\text { población acti- } \\
\text { va agraria so- } \\
\text { bre pobla- } \\
\text { ción activa }\end{array}$ \\
\hline
\end{tabular}

\begin{tabular}{|c|c|c|c|}
\hline $\begin{array}{lllllllll}\text { Vizcaya } & \ldots & \ldots & \ldots & \ldots & \ldots & \ldots & \ldots & \ldots\end{array}$ & 3 & 5 & 48 \\
\hline $\begin{array}{llllllll}\text { Guipúzcoa } & \ldots & \ldots & \ldots & \ldots & \ldots & \ldots & . .\end{array}$ & 4 & 11 & 47 \\
\hline $\begin{array}{llllllll}\text { Barcelona } & \ldots & \ldots & \ldots & \ldots & \ldots & \ldots & . .\end{array}$ & 2 & 2 & 49 \\
\hline $\begin{array}{lllllllll} & \text { Navarra } & \ldots & \ldots & \ldots & \ldots & \ldots & \ldots & \ldots\end{array}$ & 10 & 31 & 36 \\
\hline $\begin{array}{lllllllll}\text { Oviedo } & \ldots & \ldots & \ldots & \ldots & \ldots & \ldots & \ldots & \end{array}$ & 19 & 18 & 29 \\
\hline $\begin{array}{llllllll}\text { Pontevedra } & \ldots & \ldots & \ldots & \ldots & \ldots & \ldots & \ldots\end{array}$ & 20 & 33 & 24 \\
\hline $\begin{array}{lllllllll}\text { Tenerife } & \ldots & \ldots & \ldots & \ldots & \ldots & \ldots & \ldots & . .\end{array}$ & 36 & 25 & 35 \\
\hline $\begin{array}{llllllllll}\text { Lugo } & \ldots & \ldots & \ldots & \ldots & \ldots & \ldots & \ldots & \ldots & \ldots\end{array}$ & 50 & 40 & 1 \\
\hline $\begin{array}{lllllllll}\text { Orense } & \ldots & \ldots & \ldots & \ldots & \ldots & \ldots & \ldots & \ldots\end{array}$ & 23 & 42 & 2 \\
\hline $\begin{array}{lllllll}\text { Coruña, La } & \ldots & \ldots & \ldots & \ldots & \ldots & \ldots\end{array}$ & 31 & 13 & 15 \\
\hline
\end{tabular}

En los tres indicadores nos encontramos con que, por una parte, hay casos en que en algunas provincias pueden adscribirse sin dudas a los grupos extremos (así, Vizcaya, Guipúzcoa y Barcelona disponen de alta renta familiar, alta tasa de población urbana y bajo porcentaje de población activa agrícola; Lugo y Orense, por el contrario, se encontrarían en el otro extremo), pero en la mitad de los casos se trata de provincias situadas en escalones muy diversos en la lista ordinal de las provincias españolas según los valores de cada indicador: Navarra, Oviedo, Tenerife, La Coruña y Pontevedra no pueden adscribirse sin más a las provincias caracterizadas por notas claramente correlacionadas con alta participación o con «alto grado de abstención».

Sin duda, un análisis más detenido de la correlación entre grado de abstencionismo y variables de tipo sociológico podría llevarnos a conclusiones en cierto modo orientadoras. Sin embargo, cabe dejar de lado esta tarea, al disponer, para discriminar entre diversos tipos de abstencionismo, de un elemento de comparación de considerable valor: el grado de abstencionismo provincial en las elecciones de 1977.

En efecto, se puede considerar que las causas que motivaban el abstencionismo «por apatía» en 1976 (es decir, el abstencionismo del tipo I, en la

18 Fuentes: Banco de Bilbao (datos para 1973) y Estudios sociológicos sobre la situación social de España, FOESSA, Madrid, 1976. 
clasificación derivada del esquema de Flanigan) persistirian en gran parte en 1977, aun cuando factores inexistentes en la primera fecha (por ejemplo, la actuación de los partidos políticos como factor de agitación y toma de conciencia política) hubieran contribuido a disminuir el abstencionismo en la segunda. Sin embargo, los factores que determinaban la abstención «activa» de 1976 desaparecerían en gran parte en 1977. Si recordamos las condiciones en que se produjo el Referéndum de 1976, aparece claro que una parte del electorado de izquierda eligió el camino de la abstención como protesta ante una serie de condicionamientos. Pero este electorado no tenía ya motivos para actuar así en 1977, una vez que los partidos de izquierda hubieran decidido participar en la campaña.

Una comparación de los resultados electorales de 1976 y 1977 (aun recordando que se trata de consultas de distinto tipo) podría así ser útil para analizar la abstención electoral y sus componentes, al permitir separar de alguna manera los casos de provincias «apáticas» (esto es, aquellas en las que el porcentaje de abstenciones es alto en las consultas electorales o referendarias previas a 1977, y en que tal porcentaje sigue siendo alto en las elecciones de este año), provincias de abstención políticamente motivada (es decir, aquellas en que la abstención fue alta antes de 1977, pero no en este año) y provincias «manipuladas», es decir, aquellas en que en las condiciones anteriores a 1977 el electorado votó masivamente, pero en las que, en las condiciones de mayor libertad de junio de 1977 , se hizo evidente la presencia de un considerable desinterés electoral, mostrando así su «verdadero» rostro.

Para llevar a cabo esta comparación hemos procedido a situar en un gráfico, en el eje horizontal, los porcentajes provinciales de abstención en el Referéndum de 1976 y en el eje vertical, los porcentajes de abstención referidos a 1977, de tal modo que la posición de cada provincia en el gráfico viene definida por dos valores, los porcentajes de abstención en cada caso. Al objeto de separar, siquiera sea elementalmente, distintos grupos de provincias, según su comportamiento abstencionista, hemos introducido en el gráfico dos líneas: una, perpendicular al eje vertical, indica el nivel de abstención media nacional en 1977; las provincias por encima de esta línea son las que tuvieron una abstención superior a la media. Y, por otro lado, una línea perpendicular al eje horizontal, que indica la abstención media en 1976; en este caso, fueron las provincias situadas a la derecha de tal línea las más abstencionistas (Fig. 2).

Como resultado, el espacio dentro de las coordenadas queda dividido en cuatro cuadrantes:

Cuadrante I: Provincias con alta abstención en 1976 y 1977.

Cuadrante II: Provincias con baja abstención en el Referéndum de 1976, pero con alta abstención en las elecciones de 1977.

Cuadrante III: Provincias con baja abstención en 1976 y 1977. 
Figura 2. Relación entre cifras de abstención, por provincias, en 1976 y 1977

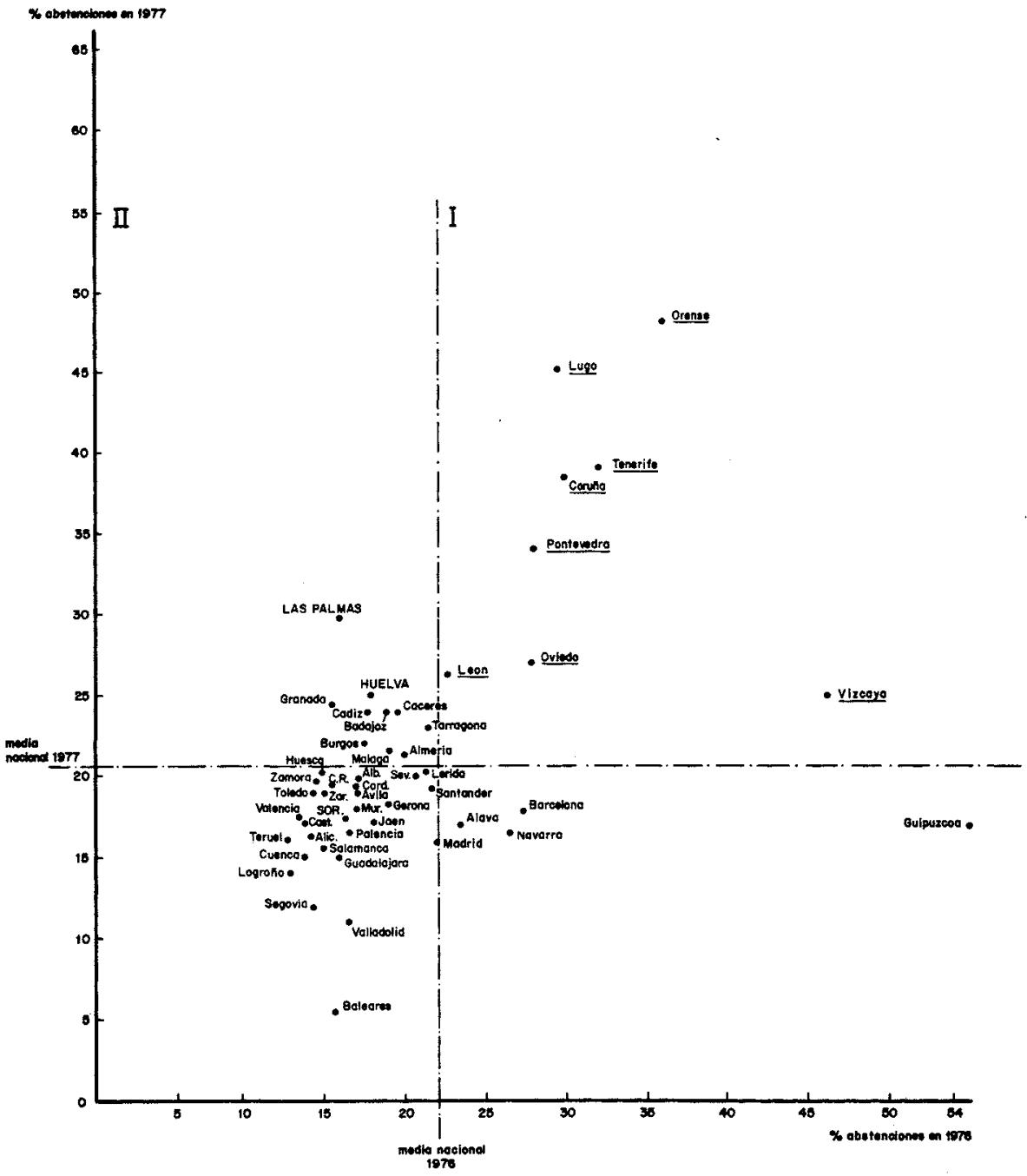


Cuadrante IV: Provincias con alta abstención en 1976 y baja abstención en $1977^{19}$.

¿Qué interpretación podía darse a la pertenencia a cada uno de estos grupos, teniendo en cuenta el esquema de Flanigan antes expuesto? Podrían considerarse al menos tres tipos definidos de provincias:

I. Provincias en que predomina un tipo de abstención derivado de la apatía política. Serían aquellas con alto nivel de abstencionismo en ambas ocasiones (1976 y 1977), es decir, aquellas en que un porcentaje de ciudadanos superior a la media nacional no se ve motivado para acudir a las urnas (o encuentran dificultades para ello que les disuaden de participar en la votación), tanto en un régimen autoritario como en un régimen de características democráticas o predemocráticas. Serían, pues, las incluidas en el

\section{MAPA I}

\section{Provincias de abstencionismo alto en 1976 y 1977}



19 Entendemos por "alta abstención" la superior a la media nacional, y por "baja abstención" la inferior a la media. Para las elecciones de 1977, a falta de resultados oficiales hasta el momento de redactarse estas líneas, hemos utilizado las cifras dadas por la prensa de Madrid; véase, sobre todo, Diario 16 (22 de julio de 1976). 


\section{MAPA II}

Provincias con alto abstencionismo en 1977 y bajo abstencionismo en 1976

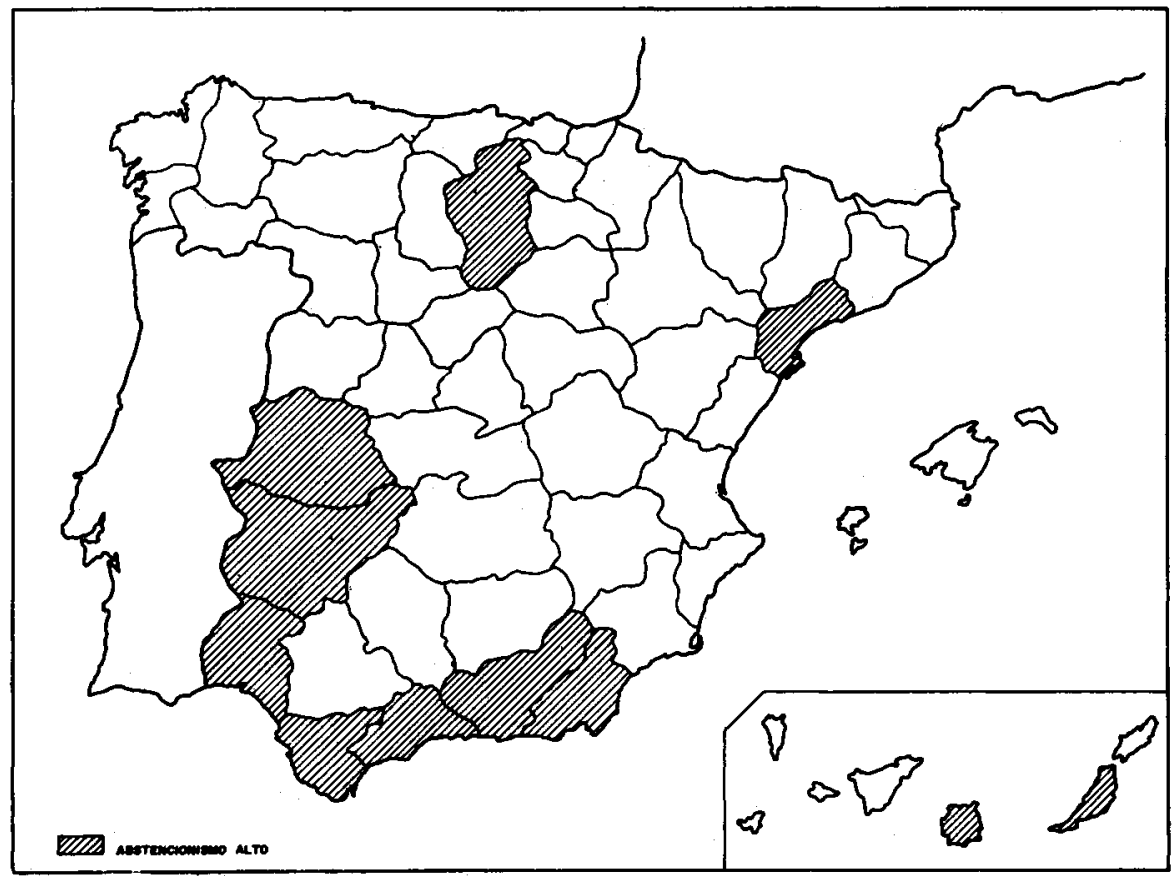

cuadrante I: La Coruña, Lugo, Orense, Pontevedra, León, Oviedo, Tenerife y Vizcaya.

Esta lista depara algunas sorpresas. En primer lugar, la aparición, al trasladar los resultados al mapa, de una amplia región con ciertas similitudes en el comportamiento abstencionista, es decir, la región N.O. de España, con seis provincias contiguas (mapa I). Lo que sugiere explicaciones de tipo socio-regional: hábitat, distribución de la población, etc.

Por otro lado destaca la presencia de la provincia de Vizcaya en este grupo, siendo una provincia con características muy distintas a las demás provincias en el cuadrante I: provincia altamente industrializada, con alta tasa de población urbana y con unos antecedentes políticos que la hace parecer todo menos «políticamente apática». Se podría conjeturar que, a pesar de todos los cambios entre 1976 y 1977, el peculiar problema vasco sigue motivando un abstencionismo de rechazo, que viene a enturbiar la clasificación general; conclusión que parece apoyada por la relativa proximidad, en el gráfico, de Guipúzcoa y Vizcaya, si bien en cuadrantes distintos. Por lo que se refiere a Tenerife, se ha configurado como altamente abstencionista en 1947, 1966, 1976 y 1977. 
II. Igualmente interesante es el caso de las provincias que se sitúan en el cuadrante superior izquierdo (II), es decir, aquellas con bajo abstencionismo en 1976 y alto abstencionismo en 1976, esto es, Burgos, Las Palmas, Cáceres, Badajoz, Cádiz, Huelva, Málaga, Granada, Almería y Tarragona. La interpretación de esta situación en el cuadrante superior izquierdo es similar a la que Flanigan da para el caso de manipulación: se trataría de provincias en que, en las condiciones de mayor libertad de 1977 se manifiesta un alto abstencionismo (hipotéticamente imputable a bajo interés por la política), mientras que en condiciones de autoritarismo político muestran altos niveles de participación.

Es interesante el que nos hallemos, en lo que se refiere a estas provincias «manipulables», con una fuerte definición regional: aparte de tres casos aislados (Burgos, Tarragona y Las Palmas), son visibles dos conjuntos regionales (mapa II), Extremadura y toda la Andalucía exterior, a lo largo de la costa.

III. Más fácil de interpretar parecen ser aquellas provincias en el cuadrante IV, es decir, aquellas provincias que habiendo obtenido un porcentaje de abstenciones superior a la media nacional en 1976, se sitúan por debajo de la misma en 1977. La explicación más simple podría ser la de que se trata de provincias en que existe una fuerte proporción de electorado de izquierda que se abstuvo (en la abstención que hemos llamado «activa» o «de rechazo») en 1976, pero no en 1977 , ocasión en que este electorado decidió intervenir en la lucha electoral. Se trata, en efecto, de Alava, Vizcaya y Navarra, Barcelona y —más matizadamente, pues en 1976 se sitúa casi en la media nacional de abstenciones- Madrid. Resultado éste que no depara muchas sorpresas, excepto la no inclusión de Vizcaya, que presenta un índice abstencionista superior al del País Vasco en general.

\section{CONCLUSIONES}

De estas observaciones, forzosamente de tipo inicial, derivadas de la comparación entre el abstencionismo electoral en dos contextos distintos (un referéndum aún en un marco autoritario en 1976 y unas elecciones en un marco de mayor libertad en 1977) se deduce, en una primera aproximación, la existencia de tres núcleos de provincias que muestrans cierta desviación respecto al comportamiento «típico» general, y que, en gran parte, coinciden con conjuntos regionales:

- Abstencionismo «de rechazo» en 1976: País Vasco (incluyendo Navarra, y con el caso excepcional de la alta abstención de Vizcaya en 1977), Madrid y Barcelona. 
- Abstencionismo apático en el N.O. de la Peninsula: Galicia, León, Oviedo. En el caso canario, alto abstencionismo, más acentuado en Tenerife. - Manipulación de votos, y abstencionismo en condiciones de libertad predemocrática, en Extremadura y la costa andaluza.

Quizá sea esta distribución regional la nota más llamativa y la que permite avanzar la hipótesis de que aun hoy las tensiones políticas, definidas regionalmente, serían características presentes, tanto en la época anterior a 1977 como en la actual, confirmando así, al menos en lo que se refiere a la conducta electoral, la existencia de subsistemas políticos regionales. El análisis de sus respectivas características sociales (así como de la estructura de sus respectivas organizaciones políticas, como partidos, redes de contactos entre élites, etc.) podría contribuir a clarificar las razones para las diferencias que separan a estos subsistemas de las condiciones políticas del resto del país. 